Abstract P2-S9.10 Table 1 Difference in socio-demographic and healthcare access and utilisation characteristics, comparing those who self-report ever testing for HIV infection to those not testing* among undoucumented Central American immigrant women in Houston, Texas, 2010

\begin{tabular}{|c|c|c|c|}
\hline Variables & Testers (\%) & Non-testers (\%) & OR (95\% Cl) \\
\hline \multicolumn{4}{|l|}{ Socio-demographics } \\
\hline \multicolumn{4}{|l|}{ Country of origin } \\
\hline Guatemala & 20.9 & 40.6 & 1.00 \\
\hline Honduras & 40.8 & 22.4 & $3.4(1.42$ to 8.38$)$ \\
\hline El Salvador & 38.3 & 37.0 & $1.96(0.88$ to 4.41$)$ \\
\hline \multicolumn{4}{|c|}{ Number of years in USA } \\
\hline Five or less & 39.9 & 68.5 & 1.00 \\
\hline Over five & 60.1 & 31.5 & 3.21 (1.62 to 6.42 ) \\
\hline \multicolumn{4}{|l|}{ Age (years) } \\
\hline $18-30$ & 38.2 & 60.8 & 1.00 \\
\hline $31-50$ & 61.8 & 39.2 & $2.59(1.32$ to 5.11$)$ \\
\hline \multicolumn{4}{|l|}{ Education } \\
\hline Sixth grade or less & 42.5 & 73.1 & 1.00 \\
\hline Over sixth grade & 57.5 & 26.9 & 3.55 (1.75 to 7.33$)$ \\
\hline \multicolumn{4}{|l|}{ Employment status } \\
\hline Unemployed & 46.7 & 54.0 & 1.00 \\
\hline Homemaker & 29.1 & 22.5 & $1.47(0.65$ to 3.41$)$ \\
\hline Employed & 24.2 & 23.5 & $1.14(0.50$ to 2.64$)$ \\
\hline \multicolumn{4}{|c|}{ Monthly household income } \\
\hline$\$ 800$ or less & 28.1 & 54.6 & 1.00 \\
\hline Over $\$ 800$ & 71.9 & 45.4 & 3.14 (1.58 to 6.25$)$ \\
\hline \multicolumn{4}{|c|}{ Healthcare access and utilisation } \\
\hline \multicolumn{4}{|c|}{ Has a healthcare provider } \\
\hline No & 38.6 & 70.7 & 1.00 \\
\hline Yes & 61.4 & 29.3 & 2.62 (1.33 to 5.22$)$ \\
\hline \multicolumn{4}{|c|}{ Saw healthcare provider, past 12 months } \\
\hline No & 39.0 & 67.0 & 1.00 \\
\hline Yes & 61.0 & 33.0 & 3.29 (1.67 to 6.55$)$ \\
\hline \multicolumn{4}{|c|}{ Has health insurance or coverage } \\
\hline No & 51.4 & 83.8 & 1.00 \\
\hline Yes & 48.6 & 16.2 & $4.86(2.17$ to 11.67$)$ \\
\hline
\end{tabular}

*Prevalence estimates are RDS-adjusted to account for difference in participants' social network size and recruitment patterns.

This seems to be due to their access to public health services through the county hospital district, which provides healthcare to all indigent residents regardless of immigration status. The association between HIV testing and regular healthcare indicates that access to public health services in this population increases the prevalence of HIV testing. Given that HIV detection among Central American immigrants is often delayed (leading to negative consequences for morbidity, mortality, and transmission), access to HIV screening is integral to HIV prevention in this population.

\section{P2-S9.11 HIV INFECTION AND VIOLENCE AGAINST MARRIED/ COHABITING WOMEN: FINDINGS FROM A NATIONAL HOUSEHOLD SURVEY IN RWANDA}

doi:10.1136/sextrans-2011-050108.397

J F Kayibanda, M Alary, R Bitera. Centre de recherche FRSO du CHA universitaire de Québec, Québec, Canada

Objectives We used the third Rwanda demographic and health survey (DHS) data to examine relationship between violence, gender attitudes and HIV prevalence among women and men in union.

Methods The third Rwanda DHS is a nationally representative household-based survey conducted in 10272 households in 2005.
Analyses were restricted to 2715 women and 2461 men who were legally married or cohabiting. HIV prevalence was the dependent variable whereas sexual risk factors, gender attitudes, emotional and domestic violence were independent variables. A face-to-face interview covered socio-demographic characteristics, sexual risk behaviour, domestic violence and gender attitude. Domestic violence was measured by questions from the Conflict Tactics Scale Questionnaire. HIV antibodies testing was performed using ELISA tests. Logistic regression was used for statistical analysis.

Results HIV prevalence was significantly higher among women who reported ever having experienced any form of emotional violence ( $4.7 \%$ vs $2.1 \% ; p=0.019)$, who reported ever been threaten by their husband or cohabiting partner ( $6.1 \%$ vs $2.3 \%$; $=0.026)$ and among those who reported that their fathers beat their mothers (3.4\% vs $1.9 \% \mathrm{p}=0.029)$. HIV prevalence was higher among men who reported that they are justified to hurt or beat their wives if they argue with them $(10.1 \%$ vs $2.9 \mathrm{p}=0.03)$. After adjustment for age, geographic area, number of lifetime partners, history of genital ulcers in the previous year, women who experienced at least one form of violence (either, emotional, inter parental, or intimate partner violence) demonstrated a much higher HIV prevalence [adjusted OR (AOR): $2.75 ; 95 \%$ CI 1.08 to 7.02$]$. Compared to those who confirmed that it is not justified for a husband to hurt or beat his wife if she argues with him, men who agreed with this statement had a fourfold higher HIV-prevalence (AOR: 4.15; 95\% CI 1.69 to 10.17).

Conclusion Violence experienced by women and the acceptance of wife beating by men are independent risk factors for HIV infection among married/cohabiting population in Rwanda. Interventions to prevent any form of violence towards women and hostile gender attitudes should be integrated into HIV programs.

\section{P2-S9.12 DESCRIPTIONS OF BEHAVIOURS BY PARTNER TYPE FOR ETHNIC MINORITY FEMALE ADOLESCENTS WITH HISTORIES OF ABUSE}

doi:10.1136/sextrans-2011-050108.398

J Collins, J D Champion. Texas Tech University, Health Sciences Center, Lubbock, USA

Background Identifying the sexual risk behaviour associated with relationship status informs researchers and clinicians concerning female adolescent conceptualisation of partnerships in the context of risk recognition, sexual boundaries, and social expectations of relationships. This study describes risk behaviour of high-risk ethnic minority female adolescents with STI and abuse histories reporting either dating one person exclusively, not currently being in relationship, or dating more than one person.

Methods African-and Mexican-American adolescent women aged $13-18$ years $(n=559)$ were enrolled in a randomised trial of a behavioural intervention. At study entry, participants completed semistructured interviews including questions addressing primary outcomes including STI infection, abuse recurrence, unintended pregnancy, sexual behaviour, substance use, and contraceptive use Descriptive, $\chi^{2}$ analyses, and t-tests for bivariate analysis of differences between groups by relationship status at study entry were conducted. Results Participants (59\%) reported dating one person exclusively, not currently involved with a partner $(29.2 \%)$, and dating more than one person (4.3\%). Participants not currently in a relationship vs those who were with one partner exclusively described more often a most recent partner who would physically harm them if she had sex with another $\operatorname{man} \chi^{2}(1, \mathrm{~N}=527)=4.51, \mathrm{p}=0.034$; having more guy friends they just have sex with $\chi^{2}(1, \mathrm{~N}=531)=7.74, \mathrm{p}<0.005$; and not having a steady relationship with their most recent partner $\chi^{2}$ $(1, N=529)=174.86, p<0.0001$. Of participants not currently in 
relationship, (30.7\%) reported they would still have sex with their most recent partner yet $95 \%$ stated they currently do not have a monogamous relationship with this partner.

Conclusions Female adolescents with abuse histories who describe relationship status as not involved or involved with more than one partner engage in more risk behaviour than those who report exclusive relationships with one partner. Explicating behaviour that occurs by relationship status has implications for interpreting and addressing risk for intervention and practice guidelines.

\section{P2-S9.13 AFRICAN- AND MEXICAN-AMERICAN ADOLESCENT WOMEN WITH STI AND A HISTORY OF ABUSE: BIOLOGICAL OUTCOME OF A RANDOMISED TRIAL OF BEHAVIOURAL INTERVENTION}

doi:10.1136/sextrans-2011-050108.399

J D Champion, J Collins. Texas Tech University, Health Science Center, Lubbock, USA

Background To implement a randomised trial of a risk-reduction intervention consisting of small group sessions, individual counselling and support groups for African- and Mexican-American adolescent women with STI and abuse history and evaluate the effects of the intervention model vs enhanced counselling for this group on STI at 6 and 12 month follow-up.

Methods Mexican- and African-American adolescent women ( $n=409,13-18$ years) with STI and a history of abuse (physical, psychological, sexual) were recruited from public-health clinics in a metropolitan area of the USA. Following enrolment participants received targeted physical exams including contraception counselling and interviews including assessments for abuse, sexual risk behaviour, substance use, contraceptive use and STI. Participants were encouraged to return for unscheduled visits as needed for counselling or suspected STI or pregnancy. Scheduled follow-up rates at $6(93 \%)$ and $12(93 \%)$ months were high Participation in risk-reduction interventions was also high (92\%). Surveillance for STI and pregnancy at off-site clinic visits was conducted and documented throughout the study.

Results At study entry, participants reported sexual (58.9\%), physical (76.8\%) and psychological (82.4\%) abuse histories, ever pregnant (45\%), currently pregnant $(18.7 \%)$, ever used birth control $(29.9 \%)$, currently in school (63.2\%), previous arrest $(56.2 \%)$, home runaway $(54.5 \%)$, any substance use $(94.1 \%)$ including marijuana $(82.6 \%)$, heroin $(13.4 \%)$, cigarettes $(74.6 \%)$, alcohol $(77.5 \%)$, cocaine $(41.1 \%)$ and benzodiazepine (45\%); forced sex $(29.1 \%)$, sex when high/out of control $(13.9 \%)$, sex with friends for benefits $(36.2 \%)$, steady partner in last 3 months (77.4\%) however off and on (31.4\%). More workshop vs control group participants reported physical abuse $(82.9 \%$ vs $71.0 \%)$, home runaway $(63.1 \%$ vs $46.4 \%)$, cigarettes $(78.9 \%$ vs $70.5 \%)$ and alcohol use ( $81.4 \%$ vs $73.8 \%)$. More control group participants were currently in school ( $68.1 \%$ vs $58.1 \%)$ and had used condoms (95.3\% vs $86.4 \%)$. These variables plus age/ethnicity were initially included in the regression model for analysis of data from 0 to 6 and $0-12$ month follow-ups. Fewer STI re-infections for intervention vs control group participants at each study interval were identified; physical abuse and substance use remained in the model.
Conclusions Behavioral interventions may reduce of STI among African-and Mexican-American adolescent women with STI and a history of abuse.

\section{P2-S9.14 A QUALITATIVE, LONGITUDINAL STUDY OF POST- DIAGNOSIS REACTIONS AMONG HSV-2 SEROLOGIC POSITIVE WOMEN}

doi:10.1136/sextrans-2011-050108.400

${ }^{1} \mathrm{~J}$ E Brand, ${ }^{2}$ B Van Der Pol. 'Marion County Health Department, Ball State University, New Palestine, USA; ${ }^{2}$ Indiana University Medical Center \& IU-Bloomington, Bloomington, USA

Background Herpes simplex virus type 2, the main cause of genital herpes, is found worldwide among populations. US National seroprevalence is estimated at $16.2 \%$, with highest rates among women (20.9\%) \& non-Hispanic blacks (39.2\%). Medical consequences of HSV-2 infection includes a two- to five-fold increased risk for HIV 1 transmission \& neonatal herpes. The advent of type-specific HSV serologic tests offers accurate methods of diagnosis for those who are asymptomatic. However, diagnosis of HSV-2 has been noted to be distressing for those who are asymptomatic \& unaware of infection. The purpose of this study is to understand social \& emotional impact of HSV-2 serodiagnosis on asymptomatic women over time.

Methods Purposeful sampling was conducted \& 28 women, newly diagnosed as HSV-2 serologic positive \& asymptomatic, were recruited from a Midwestern STD clinic \& urban community court. A series of three open-ended interviews were conducted over 6-month period. Interviews were audio recorded \& transcribed. Important areas explored: emotional \& social responses to diagnosis; motivations for (non) disclosure of HSV-2 status; exploration of sexual behaviour post-diagnosis; use of condoms \& suppressive therapy. Qualitative analysis was done using manual coding.

Results Age of participants ranged from 19 to 61 yrs. Majority were African-American (71\%) with 21\% white. No participants reported knowledge of HSV-2 status at diagnosis. Five themes emerged during analysis of first interviews: rumination \& disclosure anxiety; knowledge deficit anxiety; stigmatisation \& alteration in selfconcept; fear/apprehension regarding future; impact on sexuality \& partnering. With analysis of 6-month interviews there was an iteration of two themes-alteration in self-concept \& impact on sexuality \& partnering. Of 23 participants who completed three interviews-22\% had no plans for sex after diagnosis, 39\% never disclosed HSV-2 status to partners \& $56 \%$ never used suppressive therapy.

Conclusions Findings suggest that despite increased public information related to HSV-2, initial diagnosis remains traumatic, \& for a small percentage anxiety lingers for at least 6 months. Providers should be aware of need for written information targeted to nonclinicians \& that further follow-up should be initiated after diagnosis to reinforce learning, clarify concerns, counsel \& support. Providers should plan additional time for client integration of diagnosis, implications \& questions. 